01

\title{
Расчетная модель приемника тепловых изображений в архитектуре электронно-оптического преобразователя
}

\author{
(С) А.С. Гревцев, П.А. Золотухин, Э.А. Ильичев, Г.Н. Петрухин, А.В. Попов, Г.С. Рычков \\ Национальный исследовательский университет „Московский институт электронной техники“, \\ 124498 Зеленоград, Московская обл., Россия \\ e-mail: edil44@mail.ru, alexcoretex@gmail.com
}

Поступило в Редакцию 22 октября 2021 г.

В окончательной редакции 5 ноября 2021 г.

Принято к публикации 10 января 2022 г.

Рассмотрена инновационная конструкция и представлены результаты анализа и расчетов характеристик приемника тепловых изображений $(3-15 \mu \mathrm{m})$, выполненного в архитектуре электронно-оптического преобразователя. Для сенсорно-преобразовательного пироэлектрического узла приемника изображений рассчитаны пространственные зависимости напряженностей электрического поля и величин электрических потенциалов от спонтанной поляризации вещества пленки. Получены оценки и обсуждаются характеристики поляризации различных пироэлектрических пленок, индуцированной тепловым полем. Методом конечных элементов в программном пакете COMSOL Multiphysics рассчитаны температурные зависимости поляризационных характеристик ряда пироэлектрических пленок. Учтены возможные вклады пьезоэлектрического эффекта в картину распределения электрических потенциалов от тепловой поляризации пироэлектрических пленок. Получены оценки для предельных величин основных приборных характеристик приемника изображений.

Ключевые слова: электронно-оптический преобразователь, спонтанная поляризация, пироэлектрик, болометрические тепловизоры, пироэлектрические тепловизоры.

DOI: $10.21883 /$ JTF.2022.04.52237.270-21

\section{Введение}

Разработка конкурентоспособного и неохлаждаемого тепловизионного приемника изображений, чувствительного в спектральном диапазоне $3-15 \mu \mathrm{m}$, является актуальной задачей. Существующие в настоящее время приемники тепловых изображений можно условно объединить в три группы: фотонные - на основе узкозонных полупроводников, квантовых ям мультислойных гетероструктур, легированных полупроводников, либо диодов Шоттки; болометрические резистивные - на основе сверхпроводящих и полупроводниковых материалов; пироэлектрические - на основе эффекта поляризации сенсорно-преобразовательной пленки. За исключением тепловых приемников с сенсорами на пироэлектрических пленках, все другие нуждаются в глубоком охлаждении, вплоть до гелиевых температур [1-13].

\section{1. Современное состояние развития систем визуализации тепловых изображений}

Предельные характеристики существующих приемников тепловых изображений упомянутых классов представлены в табл. 1 и 2. В частности, в табл. 1 показаны характеристики приемников тепловых изображений с сенсорами, в основе работы которых лежат генерационно-рекомбинационные процессы, а сигнал регистрируется в виде тока неравновесных носителей.

В табл. 2 представлены характеристики тепловизионных приемников изображений с сенсорами, в основе работы которых лежат терморезистивые, емкостные и поляризационные эффекты. Также здесь представлены неохлаждаемые приемники изображений теплового диапазона, выполненные на основе сегнето- и пироэлектрических материалов.

Представленные в табл. 1 и 2 данные показывают, что приемники изображений теплового диапазона имеют существенные различия по основным предельным характеристикам. Это обусловлено отличием физических эффектов, обеспечивающих реализацию процессов регистрации и преобразования тепловых изображений их сенсорными элементами. Так, для пироэлектрических видиконов с емкостным способом считывания необходимым условием является глубокая модуляция интенсивности входного сигнала, что неизбежно приводит к росту массы и габаритов устройств, их энергозатрат. Что же касается приемников с фотонными и резистивными сенсорно-преобразовательными средами, то их эксплуатация требует глубокого охлаждения (вплоть до $1-4 \mathrm{~K}$ ).

Общим недостатком всех существующих тепловизионных систем является необходимость дополнительной привязки теплового канала изображения к местности, например, посредством использования соосносвязанного с ним оптического канала (инфракрасного либо видимого диапазона). 
Таблица 1. Основные характеристики различных конструкций фотонных приемников теплового диапазона

\begin{tabular}{c|c|c|c|c|c|c}
\hline $\begin{array}{c}\text { Тип } \\
\text { тепловизора }\end{array}$ & $\begin{array}{c}\mathscr{R}^{*}, \\
\mathrm{~A} / \mathrm{W}\end{array}$ & $\begin{array}{c}D^{* *}, \mathrm{~cm} \cdot \mathrm{Hz}^{1 / 2}, \\
\mathrm{~W}\end{array}$ & $\begin{array}{c}\text { Рабочий спектральный } \\
\text { диапазон, } \mu \mathrm{m}\end{array}$ & $\begin{array}{c}\text { Рабочая } \\
\text { температура, } \mathrm{K}\end{array}$ & $\begin{array}{c}\mathrm{NETD}^{* * *}, \\
\mathrm{mK}\end{array}$ & $\begin{array}{c}\mathrm{NEP}^{* * * *}, \\
\mathrm{fW} / \mathrm{Hz}^{1 / 2}\end{array}$ \\
\hline QWIP InP/InGaAs & $8[1], 2.9[2]$ & $7 \cdot 10^{10}[1]$ & $8[1]$ & $80[1]$ & $28[3]$ & - \\
\hline $\begin{array}{c}\text { Blocked impurity band } \\
\text { (BIB) Ge:Ga }\end{array}$ & $17[3]$ & $4 \cdot 10^{10}[4]$ & $14-140[4]$ & $1.5[3]$ & - & $0.05[5]$ \\
\hline BIB Si:Sb & $6[6]$ & $2.9 \cdot 10^{10}[4]$ & $15-40[4]$ & $5[7]$ & - & $0.056[5]$ \\
\hline BIB Si:As & $5[8]$ & $2.8 \cdot 10^{10}[4], 10^{14}[9]$ & $2-25[10]$ & $7-10[9]$ & - & $0.04[5]$ \\
\hline $\begin{array}{c}\text { Тепловизоры } \\
\text { на фотодиодах InSb }\end{array}$ & $\sim 1-2[11]$ & $10^{11}$ & $1-5[10]$ & $0.6-77[10]$ & $14.6[12]$ & $800[13]$
\end{tabular}

Пр имечание. * $\mathscr{R}$ - чувствительность тепловизора, ** D - обнаружительная способность, ***NETD - шум, эквивалентный разности температур (порог чувствительности по температуре), **** NEP — мощность, эквивалентная шуму.

Таблица 2. Основные характеристики различных конструкций тепловизионных приемников, в основу работы которых положены терморезистивные, емкостные и поляризационные эффекты

\begin{tabular}{|c|c|c|c|c|c|}
\hline Тип тепловизора & $\mathscr{R}, \mathrm{V} / \mathrm{W}$ & $D, \mathrm{~cm} \cdot \mathrm{Hz}^{1 / 2} / \mathrm{W}$ & Рабочие температуры, K & NETD, mK & $\mathrm{NEP}, \mathrm{pW} / \mathrm{Hz}^{1 / 2}$ \\
\hline $\begin{array}{c}\text { Болометрические тепловизоры } \\
\left(\text { конденсаторые, } \mathrm{VO}_{x}\right)\end{array}$ & $>1000[14]$ & $9.11 \cdot 10^{8}[15]$ & $\begin{array}{c}\text { Комнатная } \\
\text { температура }[15]\end{array}$ & $99[16]$ & $50[15]$ \\
\hline $\begin{array}{c}\text { Болометрические тепловизоры } \\
\left(\text { конденсаторны } e, \mathrm{Si}^{*}\right)\end{array}$ & $10^{6}[18]$ & $2.4 \cdot 10^{9}[18]$ & $\begin{array}{c}\text { Комнатная } \\
\text { температура [17] }\end{array}$ & $50[17]$ & $2[18]$ \\
\hline $\begin{array}{l}\text { Болометрические тепловизоры } \\
\text { (резистивные, на GaAs) }\end{array}$ & $\sim 1800[19]$ & $10^{9}-2 \cdot 10^{10}[19]$ & $150[19]$ & - & $50[20]$ \\
\hline $\begin{array}{c}\text { Болометрические тепловизоры } \\
\left(\text { резистивные, на } \mathrm{Ge}^{*}\right)\end{array}$ & $4500[21]$ & $8 \cdot 10^{11}[21]$ & $2[21]$ & - & $0.5[21]$ \\
\hline $\begin{array}{c}\text { Болометрические тепловизоры } \\
\text { (барьерные на } \mathrm{Si} / \mathrm{Au})\end{array}$ & $8 \cdot 10^{8}[22]$ & - & $4[22]$ & $0.13[22]$ & $60 \cdot 10^{-6}[22]$ \\
\hline $\begin{array}{c}\text { Пироэлектрические детекторы, } \mathrm{LiTaO}_{3} \\
\text { (емкостное считывание) }\end{array}$ & 1000 & $6 \cdot 10^{9}[23]$ & $\begin{array}{c}\text { Комнатная } \\
\text { температура }\end{array}$ & $37[24]$ & $13000[25]$ \\
\hline $\begin{array}{c}\text { Пироэлектрические тепловизоры, } \mathrm{LiNbO}_{3} \\
\text { (емкостное считывание) }\end{array}$ & $7020[26]$ & $7.76 \cdot 10^{7}[26]$ & $\begin{array}{c}\text { Комнатная } \\
\text { температура }\end{array}$ & - & $2000[26]$ \\
\hline $\begin{array}{c}\text { Пироэлектрические тепловизоры, PZT } \\
\text { (емкостное считывание) }\end{array}$ & $1000-100[27]$ & $\sim 2 \cdot 10^{9}[27]$ & $\begin{array}{c}\text { Комнатная } \\
\text { температура }\end{array}$ & - & $25[27]$ \\
\hline $\begin{array}{c}\text { Пироэлектрические тепловизоры, TGS: } \\
\text { l-аланин (емкостное считывание) }\end{array}$ & $50-300$ & $\sim 10^{9}[23]$ & $\begin{array}{c}\text { Комнатная } \\
\text { температура }\end{array}$ & - & 540 \\
\hline
\end{tabular}

Примечани е. Обозначения величин те же, что в табл. 1.

\section{2. Конструкция, физическая модель процессов и результаты расчетов}

\section{1. Конструкция, принцип работы}

Ниже предложен и обсуждается альтернативный вариант тепловизионного приемника изображений, дано схематическое изображение его конструкции (рис. 1), выполненной в архитектуре электронно-оптического преобразователя (ЭОП). Изображения „излучающего“ теплового объекта и окружающей его местности (в отраженном свете ночного неба), сформированные оптико- механическим узлом приемника, фокусируются на пироэлектрическую пленку и фотокатод соответственно. Таким образом, компактная конструкция инновационного неохлаждаемого приемника позволяет в одноканальном варианте получать изображение теплового объекта в привязке к изображению местности его расположения.

Картина изображения теплового объекта индуцирует поляризацию сенсорно-преобразовательной пироэлектрической пленки, создавая вблизи ее поверхности рельеф электрического потенциала, идентичный распределению по поверхности сенсорной пленки интенсивности теплового изображения. Оптическое изображение мест- 


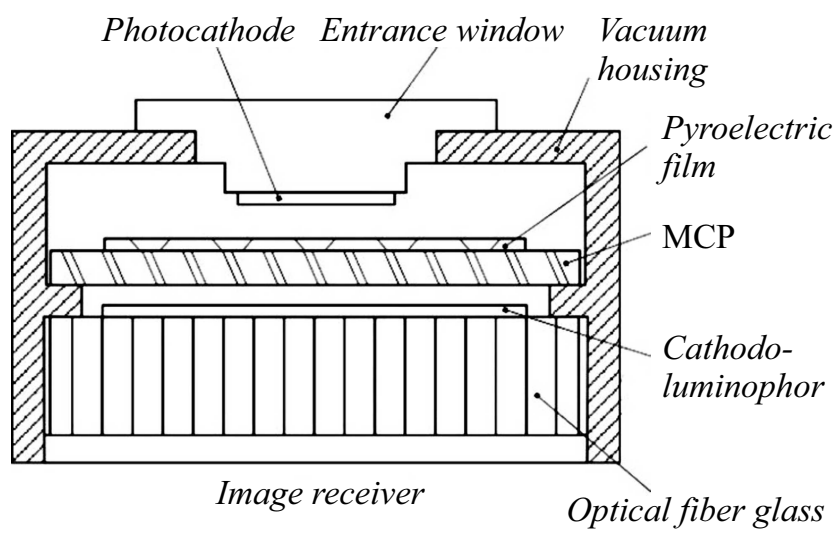

Рис. 1. Конструкция приемника тепловых изображений, выполненного в архитектуре ЭОП.

ности, сфокусированное в плоскость фотокатода, пропорционально преобразуется в изображение в фотоэлектронах. Поток фотоэлектронов направляется на поверхность пироэлектрической сенсорно-преобразовательной пленки и используется как инструмент для считывания картины распределения электрических потенциалов, тождественной картине распределения мощности излучения от теплового объекта. В результате взаимодействия с потенциальным рельефом, индуцированным тепловым полем на пироэлектрической пленке, модулированный по амплитуде поток фотоэлектронов считывается электрическим полем сквозь отверстия в пироэлектрической пленке и в несущей мембране в направлении микроканальной пластины (МКП). Умноженный в МКП поток фотоэлектронов, несущий информацию как о тепловом объекте, так и об окружающей местности, направляется на катодолюминесцентный экран и пропорционально преобразуется в фотоизображение в видимого диапазона. Последнее может быть преобразовано в цифровой формат посредством использования ПЗСмодуля, связанного иммерсионной средой с волоконнооптической пластиной выходного окна ЭОП.

\section{2. Выбор и обоснование базовых материалов}

Критериями при выборе конкретных представителей из класса пироэлектрических пленок для сенсорнопреобразовательных элементов неохлаждаемых приемников тепловых изображений должны стать температурные коэффициенты поляризации $(d \varphi / d T)$ и пространственное разрешение (малые значения коэффициента теплопроводности). Пироэлектрики обладают большими величинами спонтанной поляризации $P_{S}$, значительным пироэлектрическим коэффициентом $\left(p=d P_{S} / d T\right)$ и не нуждаются в охлаждении [28]. Однако в стандартных системах (например, в существующих видиконах) при считывании поверхностного потенциала потоком электронов наличие больших величин спонтанной поляризации $P_{S}$ может повлиять на порог чувствительности.
Поляризация вещества - результат суперпозиции проекций диполей вещества на выделенное направление, так что для коэффициента поляризации имеем (относим к единице объема):

$$
\begin{aligned}
P_{S} & =\frac{\sum_{i=1}^{n} p_{i}}{V}=\frac{\sum_{i=1}^{n} q_{i} d_{i}}{N(a+d) \Delta S}=\frac{q d n}{N(a+d) \Delta S} \\
& =\frac{q n}{\Delta S N} \frac{d}{(a+d)}=\frac{\sigma_{S} d}{a+d}=\sigma_{S},
\end{aligned}
$$

где $n$ - общее количество диполей, $N$ - количество дипольных слоев, $d-$ плечо диполя, $a-$ расстояние между дипольными слоями, $\Delta S-$ площадь пленки, $\sigma_{s}-$ поверхностная плотность заряда.

Таким образом, коэффициент спонтанной поляризации пироэлектрической пленки $\left(P_{S}\right)$ будет соответствовать плотностям поверхностного заряда $\left(\sigma_{-}\right.$и $\left.\sigma_{+}\right)$на внешних поверхностях дипольного слоя, в том числе и для случая „толстых“ пленок. Это позволяет брать информацию о поверхностном заряде (а значит о коэффициенте поляризации) из табличных данных о $P_{S}$ для любого конкретного пироэлектрика.

\section{3. Функциональные зависимости напряженности и потенциалов электрического поля от пироэлектрической пленки}

В силу рассуждений (1), расчеты электрического поля и электрического потенциала над поверхностью пироэлектрической пленки выполним в рамках модели объекта в виде совокупности двух противоположно заряженных плоскостей радиусом $R$, находящихся друг от друга на расстоянии, равном плечу диполя $d$. При этом, как выше было показано, информация о поверхностной плотности заряда в пленке может быть взята из таблиц в виде $P_{S}$.

Методика получения функциональной зависимости напряженности электрического поля для любого расстояния $Z$ от фрагмента $d S$ верхней заряженной плоскости дипольного слоя, графически иллюстрируется рис. 2 :

$$
d E_{\text {upper plane }}=\frac{d q}{4 \pi \varepsilon_{0}} \frac{1}{r_{-}^{2}} \cos (\theta),
$$

где $\mathbf{r}_{-}$- радиус-вектор от точки $z$ до выделенной элементарной площади $d S$ с координатами $(\varphi, r)$ на верхней плоскости с зарядом $d q, \varepsilon_{0}$ - диэлектрическая постоянная, $\cos (\theta)$ - косинус угла между векторами $\mathbf{r}_{-}$и $\mathbf{r}$.

Для связи заряда $d q$ локализованного на площади $d S$ верхней плоскости дипольного слоя с коэффициентом поляризации пленки, в цилиндрических координатах имеем

$$
d q=-\sigma d S=-P_{S} d S=-P_{S} J(\varphi, z) d \varphi d r=-P_{S} r d \varphi d r,
$$




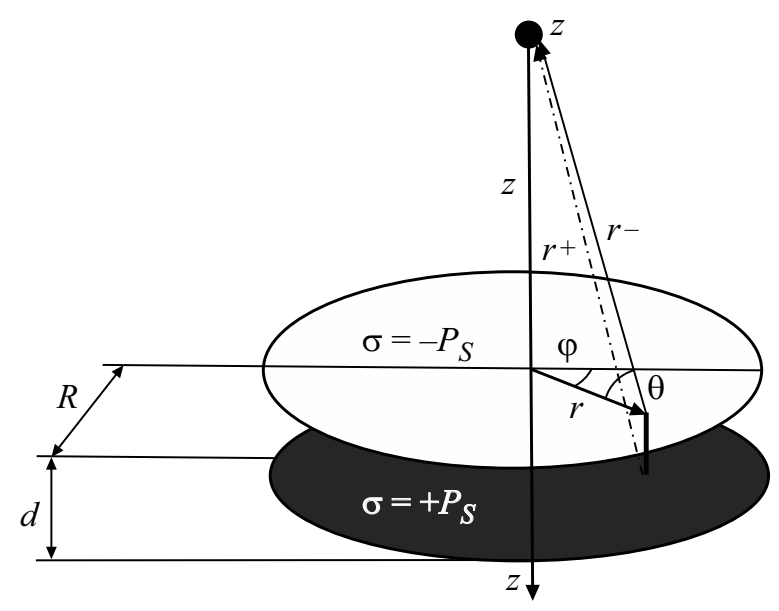

Рис. 2. Модель дипольного слоя для расчета поверхностного потенциала в любой точке $z$.

где $d S$ - элемент площади верхней пластины, $J(\varphi, \mathbf{r})-$ якобиан в цилиндрических координатах. Поэтому, представив (2) в виде

$$
\begin{aligned}
d E_{\text {upper plane }}= & -\frac{P_{S} r d \varphi d r}{4 \pi \varepsilon_{0}} \frac{1}{r_{-}^{2}} \cos (\theta)=-\frac{P_{S} r d \varphi d r}{4 \pi \varepsilon_{0}} \\
& \times \frac{1}{z^{2}+r^{2}} \frac{z}{\sqrt{z^{2}+r^{2}}},
\end{aligned}
$$

для координатной зависимости напряженности электрического поля от верхней плоскости дипольного слоя радиуса $R$,получаем (см. Приложение 1 ) соотношение (3):

$$
E_{\text {upper plane }}=\left.\left(\frac{P_{S}}{2 \varepsilon_{0}} \frac{z}{\sqrt{z^{2}+r^{2}}}\right)\right|_{0} ^{R}=-\frac{P_{S}}{2 \varepsilon_{0}}\left(1-\frac{z}{\sqrt{z^{2}+R^{2}}}\right) .
$$

Аналогично (3) получаем координатную зависимость напряженности электрического поля от несущей положительный заряд нижней плоскости дипольного слоя:

$$
E_{\text {lower plane }}=\frac{P_{S}}{2 \varepsilon_{0}}\left(1-\frac{z+d}{\sqrt{(z+d)^{2}+R^{2}}}\right) .
$$

Суперпозиция полей (3) и (4) дает координатную зависимость полного поля от дипольного слоя $E_{\text {diplayer: }}$ :

$$
\begin{aligned}
E_{\text {dip layer }}= & E_{\text {upper plane }}+E_{\text {lower plane }}=\frac{P_{S}}{2 \varepsilon_{0}}\left(\frac{z}{\sqrt{z^{2}+R^{2}}}\right. \\
& \left.-\frac{z+d}{\sqrt{(z+d)^{2}+R^{2}}}\right) .
\end{aligned}
$$

Знание величины напряженности электрического по-

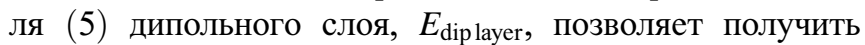
выражение для электрического потенциала в любой координатной точке $z$. Координатную зависимость электрического потенциала от расстояния $(z)$ до дипольного слоя получаем (см. Приложение 2), интегрируя выражение (5):

$$
\varphi(z)=\frac{P_{S}}{2 \varepsilon_{0}}\left(\sqrt{(z+d)^{2}+R^{2}}-\sqrt{z^{2}+R^{2}}-d\right) .
$$

Заметим, что из (6) при $z \rightarrow \infty$ получаем $\varphi(z)=0$, что является разумным граничным условием.

При решении прикладных задач важны знания не только предельных значений основных параметров изделия, но и их аппроксимации. Ниже представлены результаты аппроксимации координатных зависимостей электрических потенциалов и напряженностей электрического поля от поляризации дипольного слоя, выполненные в приближениях $R \gg z$ и $z \gg R$.

\section{Случай $R \gg z$}

Представив выражение (5) в виде

$$
E=\frac{P_{S}}{2 \varepsilon_{0}} \frac{1}{\sqrt{z^{2}+R^{2}}}\left(z-\frac{z+d}{\sqrt{1+\frac{2 z d+d^{2}}{z^{2}+R^{2}}}}\right)
$$

после ряда преобразований, получаем (см. Приложение 3):

$$
\begin{aligned}
E & =\frac{P_{S}}{2 \varepsilon_{0}} \frac{1}{R}\left(z-\frac{2(z+d) R^{2}}{2 R^{2}+2 z d+d^{2}}\right) \\
& =-\frac{P_{S}}{2 \varepsilon_{0}} \frac{2(z+d) R}{2 R^{2}+2 z d+d^{2}}=-\frac{P_{S}}{2 \varepsilon_{0}} \frac{(z+d)}{R} .
\end{aligned}
$$

Отсюда при $R \gg d \gg z$

$$
E=-\frac{P_{S}}{2 \varepsilon_{0}} \frac{d}{R}
$$

при $R \gg z \gg d$

$$
E=-\frac{P_{S}}{2 \varepsilon_{0}} \frac{z}{R}
$$

Аналогично из уравнения (6) для $R \gg z$ получаем аппроксимацию для электрического потенциала (см. Приложение 3):

$$
\varphi(z)=\frac{P_{S}}{2 \varepsilon_{0}} R\left(\frac{2 z d+d^{2}}{2 R^{2}}-\frac{d}{R}\right) .
$$

Для случая $2 z d \gg d^{2}$ выражение (9) принимает вид

$$
\varphi\left(z_{0}\right)=\frac{P_{S}}{2 \varepsilon_{0}}\left(\frac{z d}{R}-d\right)
$$

\section{Случай $z \gg R$}

Преобразуем выражение (4) к виду

$$
E_{\text {upper plane }}=-\frac{P_{S}}{2 \varepsilon_{0}}\left(1-\frac{z}{z \sqrt{1+\frac{R^{2}}{z^{2}}}}\right) .
$$


Разлагая (11) в ряд Тейлора, получаем

$E_{\text {upper plane }}=-\frac{P_{S}}{2 \varepsilon_{0}}\left(1-\frac{z}{z\left(1+\frac{R^{2}}{2 z^{2}}\right)}\right)=-\frac{P_{S}}{2 \varepsilon_{0}}\left(\frac{R^{2}}{2 z^{2}+R^{2}}\right)$.

откуда при $z \gg R$ получаем

$$
E_{\text {upper plane }}=-\frac{P_{S}}{4 \varepsilon_{0}} \frac{R^{2}}{z^{2}} .
$$

Аналогично в рамках указанного приближения $(z \gg R)$ находим напряженность электрического поля

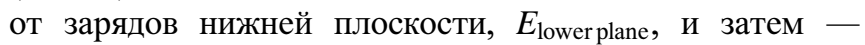
функциональную зависимость полного электрического поля от дипольного слоя:

$$
\begin{aligned}
E_{\text {upperplane }}+E_{\text {lower plane }} & =\frac{P_{S} R^{2}}{4 \varepsilon_{0}}\left(\frac{1}{(z+d)^{2}}-\frac{1}{z^{2}}\right) \\
& =\frac{P_{S} R^{2}}{4 \varepsilon_{0}}\left(\frac{-2 z d-d^{2}}{z^{2}(z+d)^{2}}\right),
\end{aligned}
$$

откуда в выбранных приближениях следует

$$
E=-\frac{P_{S} R^{2}}{2 \varepsilon_{0}} \frac{d}{z^{3}}
$$

При получении координатной зависимости электрического потенциала в случае $z \gg R$, интегрируя выражение (13), получаем (см. Приложение 4):

$$
\varphi(z)=-\frac{P_{S} R^{2}}{4 \varepsilon_{0}} \frac{d}{z^{2}} .
$$

Таблица 3. Итоговая таблица аппроксимаций потенциалов и напряженностей электрического поля дипольного слоя

\begin{tabular}{c|c|c|c}
\hline \multicolumn{2}{c|}{$z \gg R$} & \multicolumn{2}{|c}{$R \gg z$ и $d \gg z$} \\
\hline$\varphi(z)$ & $E(z)$ & $\varphi(z)$ & $E(z)$ \\
$(14)$ & $(13)$ & $(10)$ & $(7)(8)$ \\
\hline$-\frac{P_{S} R^{2}}{4 \varepsilon_{0}} \frac{d}{z^{2}}$ & $-\frac{P_{S} R^{2}}{2 \varepsilon_{0}} \frac{d}{z^{3}}$ & $\frac{P_{S} d}{2 \varepsilon_{0}}\left(\frac{z}{R}-1\right)$ & $-\frac{P_{S}}{2 \varepsilon_{0}} \frac{d}{R}$ или $-\frac{P_{S}}{2 \varepsilon_{0}} \frac{z}{R}$
\end{tabular}

Итоговые результаты аппроксимации потенциалов и полей представлены в табл. 3.

\section{4. Расчеты и графическое представление координатных зависимостей напряженности и потенциалов электрических полей}

Полученные выше функциональные зависимости были использованы для численных расчетов распределений напряженностей электрических полей и электрических потенциалов в окружающем сенсорные пленки пространстве. Необходимые для расчетов параметры использованных пироэлектрических пленок представлены в табл. 4.

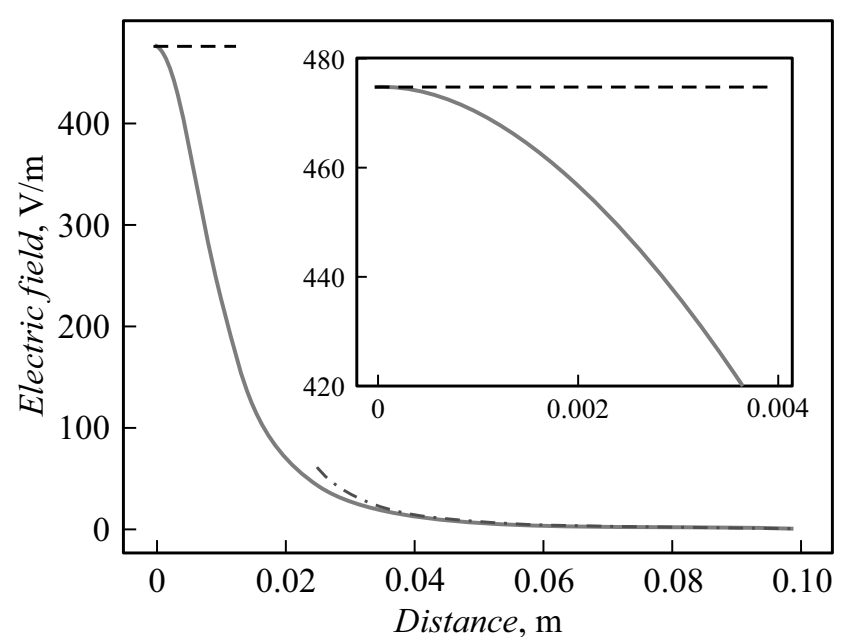

Рис. 3. Зависимость от расстояния напряженности электрического поля от дипольного слоя в любой точке $z$ для PZT-5Н.

Форма и геометрические размеры пироэлектрической пленки и несущей ее сетчатой полупроводниковой мембраны выбирались исходя из формы и размеров вакуумно-плотного корпуса приемника изображений: толщина сетчатой полупроводниковой мембраны $\sim 30 \mu \mathrm{m}$, толщина пироэлектрической пленки $\sim 0.1-1.0 \mu \mathrm{m}$, радиус пленки и $\left(\mathrm{Si} / \mathrm{SiO}_{2}\right)$ мембраны $R \approx 12.5 \mathrm{~mm}$.

Таблица 4. Основные параметры пироэлектрических пленок

\begin{tabular}{c|c|c}
\hline Материал & $\begin{array}{c}\text { Пироэлектрический } \\
\text { коэффициент } \\
p=d P_{S} / d T, \frac{\mu \mathrm{C}}{\mathrm{m}^{2} \mathrm{~K}}\end{array}$ & $\begin{array}{c}\text { Модуль вектора } \\
\text { спонтанной поля- } \\
\text { ризации } P_{S}, \frac{\mathrm{C}}{\mathrm{m}^{2}}\end{array}$ \\
\hline $\begin{array}{c}\text { PZT-5H } \\
(\text { Цирконат-тита- } \\
\text { нат свинца) }\end{array}$ & $-450[29]$ & $0.35[29]$ \\
\hline $\begin{array}{c}\text { TGS с } l \text {-аланином } \\
\text { (Триглицинсульфат) }\end{array}$ & $-400[29]$ & $0.03[29]$ \\
\hline $\begin{array}{c}\text { ВSN50 (Ниобат } \\
\text { барий-стронций) }\end{array}$ & $-550[29]$ & $0.065[28]$
\end{tabular}

Ниже (рис. 3-8) представлены расчетные графики распределения в пространстве электрических потенциалов и напряженностей электрического поля. Там же (пунктирными линиями) указаны их аппроксимации.

\section{3. Анализ поведения процессов поляризации, индуцированных в пироэлектрических пленках тепловым полем}

Выполним оценки изменений величин поляризации и электрических потенциалов в пространстве, окружающем пироэлектрические пленки, вызванных воз- 


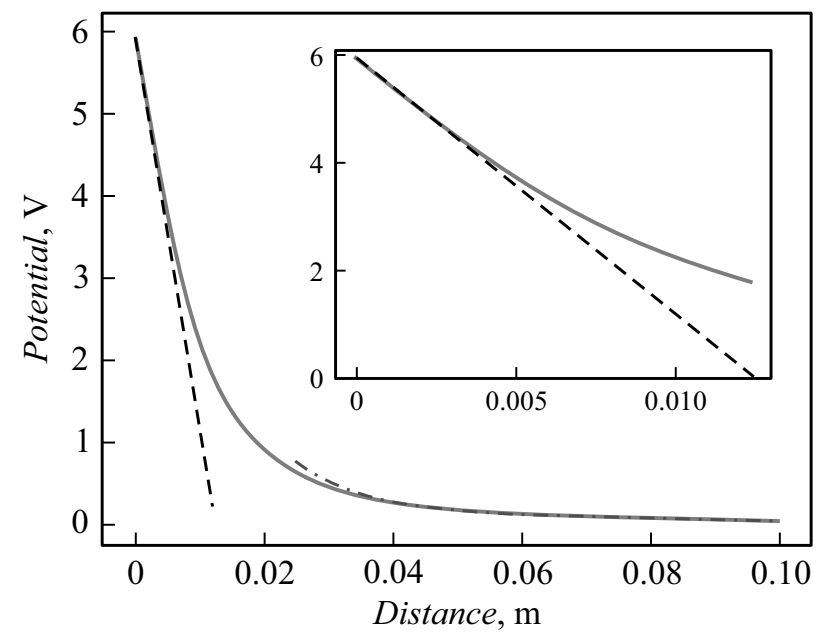

Рис. 4. Зависимость от расстояния электрического потенциала от дипольного слоя в любой точке $z$ для РZT-5Н.

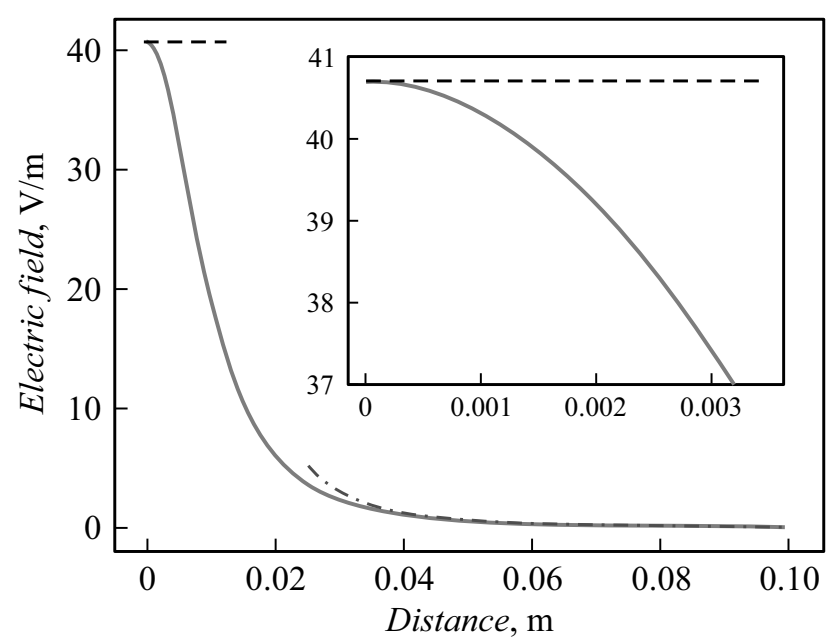

Рис. 5. Зависимость от расстояния напряженности электрического поля от дипольного слоя в любой точке $z$ для TGS c $l$-аланином.

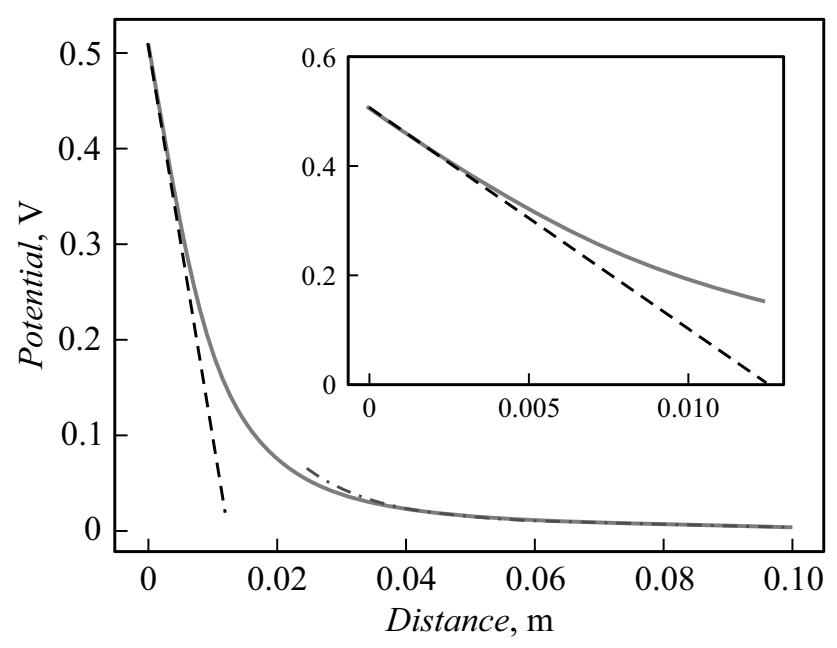

Рис. 6. Зависимость от расстояния электрического потенциала от дипольного слоя в любой точке $z$ для TGS с $l$-аланином.

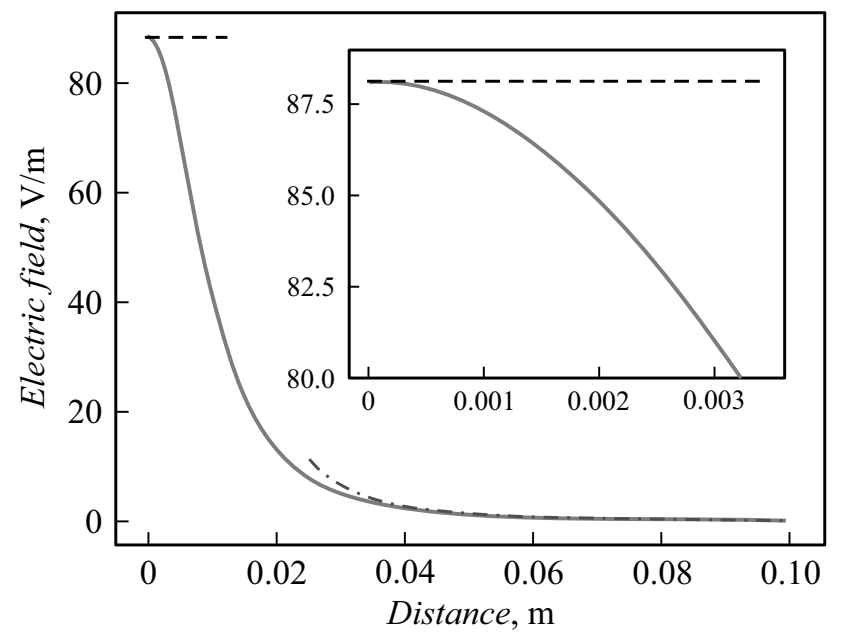

Рис. 7. Зависимость от расстояния напряженности электрического поля от дипольного слоя в любой точке $z$ для BSN50.

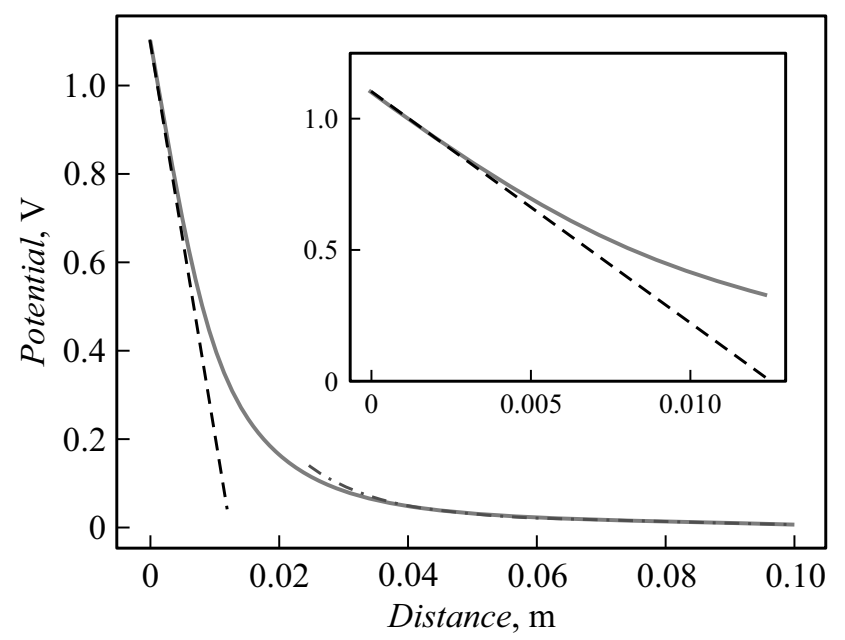

Рис. 8. Зависимость от расстояния электрического потенциала от дипольного слоя в любой точке $z$ для BSN50.

действием теплового поля. Пусть исходная структура представляет собой кремниевую мембрану радиусом $12.5 \mathrm{~mm}$ и толщиной $30 \mu \mathrm{m}$, на которой последовательно расположены слои оксида кремния толщиной $\sim 1 \mu \mathrm{m}$ и пироэлектрической пленки толщиной $\sim 1 \mu \mathrm{m}$.

Динамику нагрева приборной структуры определим, используя уравнение теплового баланса:

$$
\rho C_{\rho} \frac{d T}{d t}+\nabla\left(\mathbf{q}+\mathbf{q}_{r}\right)=\gamma P .
$$

Здесь $\mathbf{q}$ - потери потока тепловой мощности за счет теплопроводности структуры

$$
\mathbf{q}=-G \nabla T,
$$

$G$ - коэффициент теплопроводности многослойной структур, $\rho-$ плотность, $C_{\rho}-$ теплопроводность, $\mathbf{q}_{r}-$ потери потока тепловой мощности, связанные с излучением (оцениваем в приближении Стефана-Больцмана)

$$
\mathbf{q}_{r} \mathbf{n}=\gamma \sigma\left(T^{4}-T_{\text {initial }}^{4}\right)
$$


$\mathbf{n}-$ единичный вектор нормали к поверхности исследуемой структуры, $T_{\text {initial }}-$ начальная температура, $T-$ текущая температура сенсорно-преобразовательного слоя, $\sigma$ - постоянная Стефана-Больцмана, $\gamma-$ коэффициент черноты поверхности сенсорной пленки.

Таблица 5. Параметры материалов, используемых при расчете индуцированной поляризации пироэлектрических пленок, расположенных на кремниевой мембране

\begin{tabular}{c|c|c|c|c|c}
\hline Материал & $C_{p}, \frac{\mathrm{J}}{\mathrm{kg} \cdot \mathrm{K}}$ & $G_{\text {th }}, \frac{\mathrm{w}}{\mathrm{m} \cdot \mathrm{K}}$ & $\rho, \frac{\mathrm{kg}}{\mathrm{m}^{3}}$ & $\gamma$ & $p, \frac{\mu \mathrm{C}}{\mathrm{m}^{2} \cdot \mathrm{K}}$ \\
\hline SBN50 (Ниобат бария-стронция) & $440[30]$ & $1.8[31]$ & $5300[31]$ & $0.88[32]$ & $-400[36]$ \\
\hline PZT-5H (Цирконат-титанат свинца) & $350[33]$ & 0.14 & 7500 & $1[34]$ & $-450[36]$ \\
\hline TGS $l$-alanine (Триглицинсульфат) & $1352[31]$ & $1[31]$ & 1700 & $1[35]$ & $-550[36]$ \\
\hline $\mathrm{Si}$ & 700 & 130 & 2329 & 0.2 & - \\
\hline $\mathrm{SiO}_{2}$ & 703 & 0 & 2203 & 0.2 & -
\end{tabular}

При расчетах нами использовалась модель теплового сопротивления (графически представлена на рис. 9, где мощность теплового потока (16) представляется в виде плотности тока, проходящего через цепь последовательно соединенных тепловых сопротивлений. Таким образом, формально значение плотности теплового потока можно представить в виде отношения разности температур верхнего и нижнего слоев пленки к сумме тепловых сопротивлений мультислойной дипольной структуры.

Параметры, характеризующие рассматриваемые пироэлектрические материалы, представлены в табл. 5, плотность мощности теплового потока, падающего на сенсорно-преобразовательную пленку, допустим равной $0.21 \mu \mathrm{W} / \mathrm{cm}^{2}$ (полная мощность, приходящая на пленку, $\sim 1 \mu \mathrm{W})$, а его длительность - примерно равной одной секунде.

Ниже (рис. 10) представлены результаты расчетов динамики изменений температуры пленки, выполненные в рамках упомянутой модели (рис. 9) с использованием уравнений $(15)-(17)$.

При расчетах поляризации пленки (рис. 11), индуцированной тепловым полем, воспользуемся уравнением (18) и полученными результатами изменений во

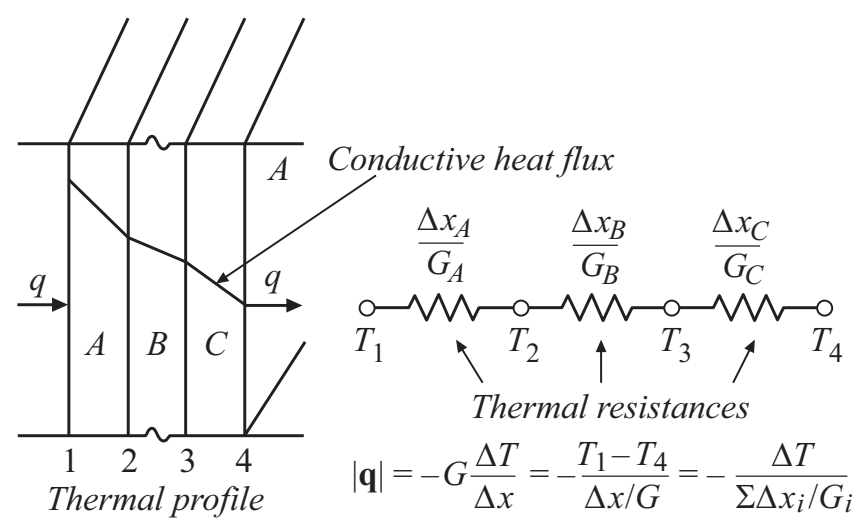

Рис. 9. Модель теплового сопротивления, используемая при расчете мощности теплового потока.

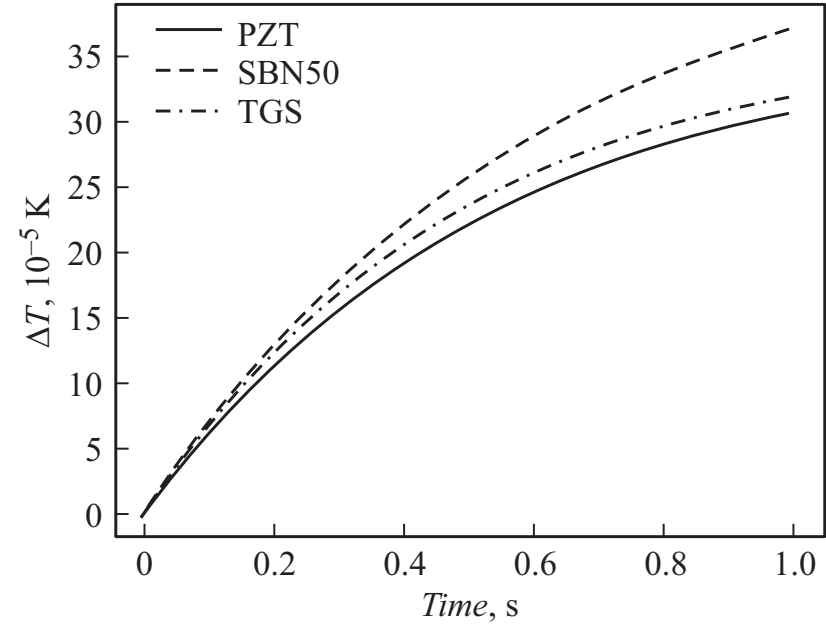

Рис. 10. Временна́я характеристика изменений температуры различных пироэлектрических пленок, индуцированных падающим потоком теплового излучения.

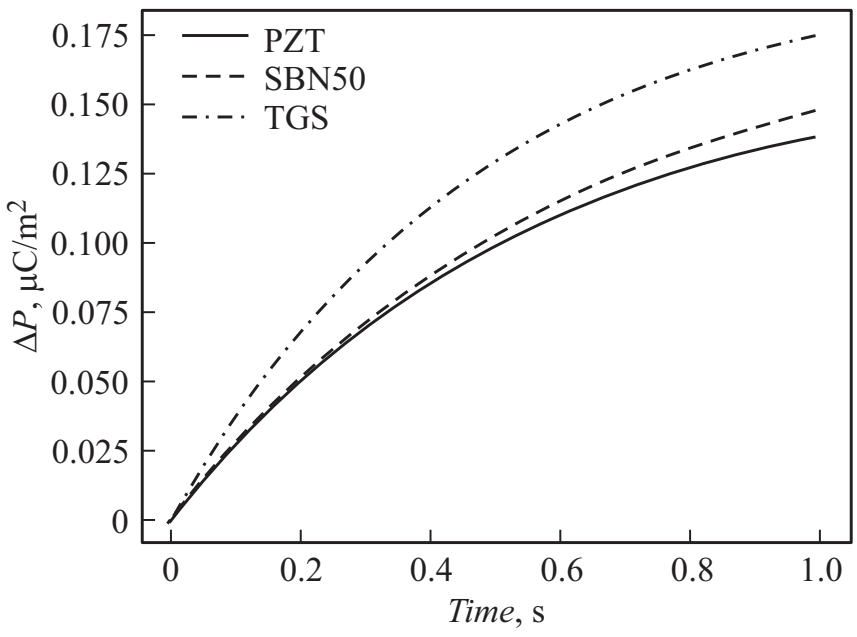

Рис. 11. Временна́я характеристика изменений поляризации различных пироэлектрических пленок, индуцированных падающим потоком теплового излучения. 


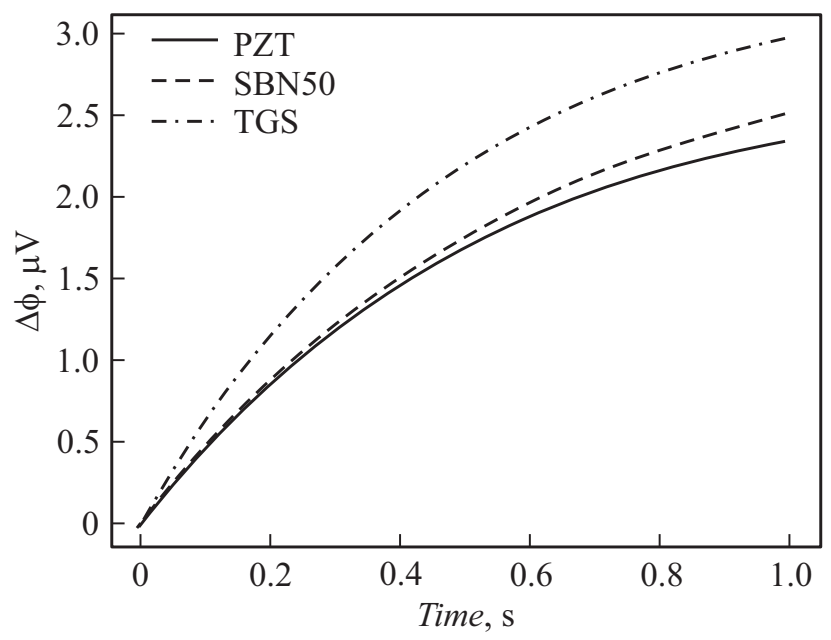

Рис. 12. Временна́я характеристика изменений поверхностного потенциала $(z \approx 0)$ для различных пироэлектрических пленок, индуцированных изменением вектора поляризации.

времени температуры пироэлектрика (рис. 10):

$$
\Delta P_{S}=p \Delta T=p\left(T_{\text {initial }}-T(t)\right),
$$

где $T_{\text {initial }}$ - начальная температура, $p$ - пироэлектрический коэффициент (табл. 5), $\Delta P_{S}$ - изменение вектора поляризации.

\section{4. Динамика изменений величин электрических потенциалов, индуцированных воздействием на пироэлектрические пленки теплового поля}

Используя уравнение (19) и временные характеристики изменений поляризации $\Delta P_{S}(t)$ (рис. 11), можно получить для фиксированной тепловой мощности (см. Приложение 2) координатную зависимость величины электрического потенциала от величины поляризации, индуцированной тепловым полем, а затем рассчитать электрический потенциал на любом расстоянии $z$ от поверхности пленки в любой момент времени:

$$
\Delta \phi(z)=\frac{\Delta P_{S}}{2 \varepsilon_{0}}\left(\sqrt{(z+d)^{2}+R^{2}}-\sqrt{z^{2}+R^{2}}-d\right) .
$$

В частности, ниже для случая $z \approx 0$ и плотности мощности $0.21 \mu \mathrm{W} / \mathrm{cm}^{2}$ представлены зависимости изменений во времени поверхностного электрического потенциала $\Delta \phi(t)$ для различных пироэлектрических пленок (рис. 12).

\section{5. Оценка возможного влияния упругих деформаций на индуцированные тепловым полем в пироэлектрической пленке электрические потенциалы}

В обсуждаемой конструкции приемника тепловых изображений пироэлектрическая пленка расположена на оксидированной поверхности (для минимизации тепловых потерь за счет теплопроводности) тонкой кремниевой мембраны. Из-за существенного различия между коэффициентами температурного расширения отдельных слоев гетероструктуры, из-за пьезоэлектрического эффекта возможны искажения индуцированной тепловым полем картины распределения электрических потенциалов. При расчетах будем пренебрегать вкладом со стороны оксидного покрытия в результирующие упругие напряжения (мотивацию см. в табл. 2). Моделирование искажений картины распределения электрических потенциалов на поверхности пироэлектрической пленки при изменениях температуры за счет пьезоэффекта выполнено на конкретных материалах (табл. 6). Логика и процедура расчетов представлена в Приложении 5 .

Таблица 6. Коэффициенты теплового расширения используемых материалов

\begin{tabular}{c|c}
\hline Материал & $\alpha, \frac{10^{-6}}{\mathrm{~K}}$ \\
\hline $\mathrm{Si}$ & 2.6 \\
\hline $\mathrm{SiO}_{2}$ & 0.55 \\
\hline $\mathrm{PZT}-5 \mathrm{H}$ & 3.6
\end{tabular}

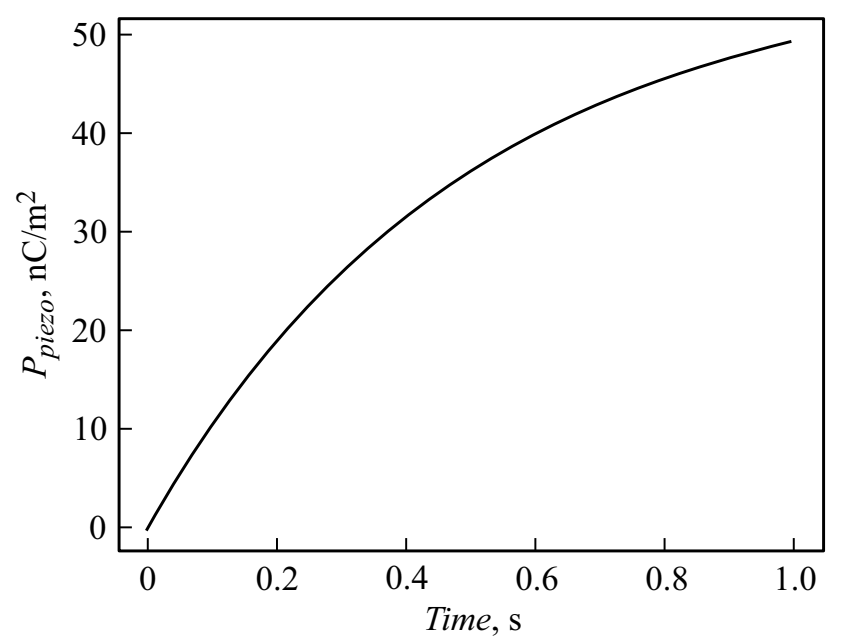

Рис. 13. Временна́я характеристика пьезоэлектрической поляризации $(z \approx 0)$ пироэлектрического образца, связанная с деформацией мультислойной структуры за счет теплового расширения. 


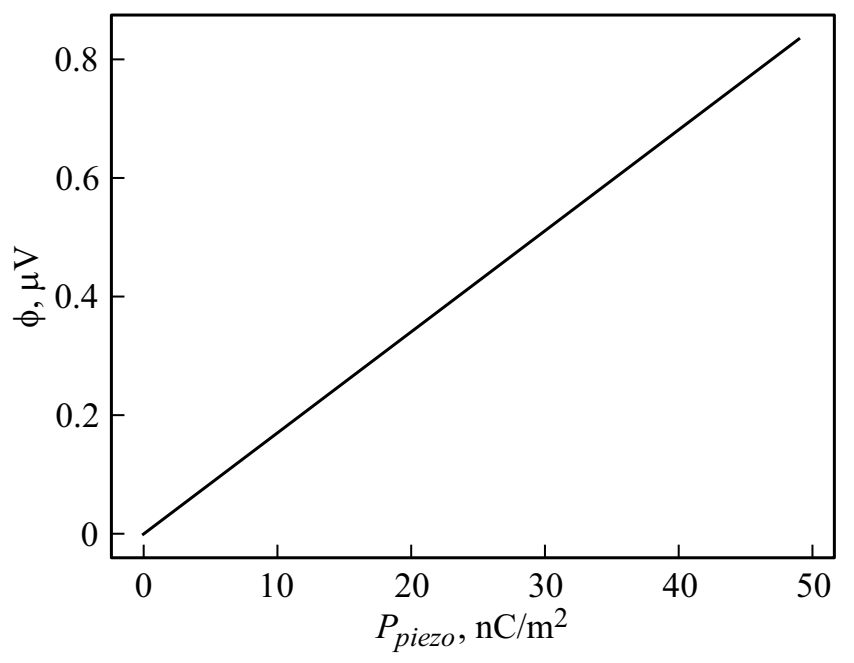

Рис. 14. Зависимость поверхностного потенциала пироэлектрического образца от величины пьезоэлектрической поляризации.

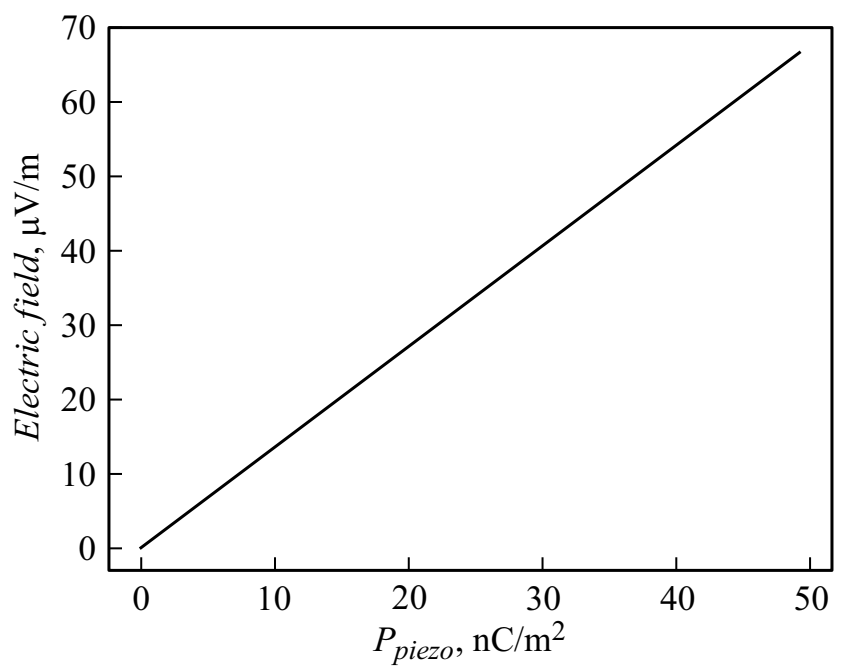

Рис. 15. Зависимость напряженности электрического поля пироэлектрического образца от величины пьезоэлектрической поляризации.

Проведенные расчеты в программном пакете COMSOL Multiphysics позволили получить графики усредненных величин электрической поляризации, электрического потенциала и напряженности электрического поля на поверхности пироэлектрика, вызванные только деформациями. Результаты расчетов представлены на рис. 13-15.

Подставляя максимальное значение для величины поляризации пироэлектрического материала (она составила $\left.P_{\text {piezo }}=50 \frac{n C}{m^{2}}\right)$ в уравнение $(19)$, получаем оценку для величины поверхностного потенциала, индуцированного пьезоэлектрическим эффектом. Она оказывается равной $\sim \Delta \phi=0.8 \mu \mathrm{V}$ при длине плеча дипольного слоя $d=0.3 \mathrm{~nm}$.

\section{6. Расчетные данные характеристики сенсорно-преобразовательных пироэлектрических пленок, интегрированных в конструкцию инновационного приемника тепловых изображений}

Результаты оценок предельных характеристик приемников тепловых изображений с конкретными представителями сенсорных пироэлектрических пленок, представлены в табл. 7. Процедура расчетов представлена в Приложении 6.

Таблица 7. Результаты расчетов поляризации и поверхностного потенциала для различных пироэлектрических образцов

\begin{tabular}{c|c|c|c|c}
\hline Материал & $\begin{array}{c}D, \mathrm{~cm} \sqrt{H z} / \mathrm{W} \\
S=16 \times 16 \mu \mathrm{m}\end{array}$ & $\begin{array}{c}\mathscr{R}, \\
\mathrm{W}\end{array}$ & $\begin{array}{c}\mathrm{NEP}, \\
\mathrm{nW} / \sqrt{H z}\end{array}$ & $\begin{array}{c}V_{N}, \\
\mathrm{nV}\end{array}$ \\
\hline SBN50 & $10^{6}$ & 3.7 & 17.8 & 178 \\
\hline PZT-5H & $8.7 \cdot 10^{5}$ & 3.1 & 17.8 & 178 \\
\hline $\begin{array}{c}\text { TGS с добавлением } \\
\text { l-аланинаа }\end{array}$ & $2.84 \cdot 10^{5}$ & 3.2 & 56.3 & 563
\end{tabular}

Примечание. Обозначения величин те же, что в табл. 1.

\section{Заключение}

Рассмотрена одноканальная инновационная конструкция приемника тепловых изображений $(3-15 \mu \mathrm{m})$, выполненная в архитектуре ЭОП. Обсуждаются процессы спонтанной и индуцированной тепловым полем поляризации пироэлектрической сенсорно-преобразовательной пленки. Тепловым сенсором-преобразователем выступает пироэлектрическая пленка. Считывание информации о пространственном распределении электрического потенциала, индуцированного тепловым изображением вблизи поверхности пироэлектрической пленки, осуществляется потоком фотоэлектронов в режиме „на прострел“ сквозь отверстия в $\mathrm{Si} / \mathrm{SiO}_{2}$-мембране. Этот поток фотоэлектронов формируется фотокатодом, расположенным на торце входного окна, и несет информацию о местности в отраженном свете ночного неба в ближнем ИК диапазоне $(0.8-1.1 \mu \mathrm{m})$. Результаты анализа и расчетов функциональных зависимостей напряженности электрического поля и величин электрических потенциалов от характеристик спонтанной поляризации пленки представлены в виде графиков и таблиц.

Для ряда пироэлектрических пленок получены оценки сравнительной эффективности пропорционального преобразования проецируемых на пленку тепловых полей в картину распределений электрических потенциалов. Учтены возможные паразитные вклады пьезоэлектрических эффектов со стороны биморфной структуры (пироэлектрическая пленка/кремниевая мембрана) в картину 
распределения электрических потенциалов. Получены оценки для предельных величин основных характеристик сенсорно-преобразовательного узла приемника тепловых изображений.

\section{Приложение 1}

Для напряженности поля верхней плоскости имеем

$$
E_{\text {upper plane }}=-\frac{P_{S} z}{4 \pi \varepsilon_{0}} \int_{0}^{R} \int_{0}^{2 \pi} \frac{r}{\left(z^{2}+r^{2}\right)^{3 / 2}} d r d \varphi
$$

отсюда

$$
E_{\text {upper plane }}=-\frac{P_{S} z}{2 \varepsilon_{0}} \int_{0}^{R} \frac{r}{\left(z^{2}+r^{2}\right)^{3 / 2}} d r
$$

и, сделав замену переменных, получаем

$$
E_{\text {upper plane }}=-\frac{P_{S} z}{4 \varepsilon_{0}} \int_{0}^{R} \frac{d\left(r^{2}+z^{2}\right)}{\left(z^{2}+r^{2}\right)^{3 / 2}} .
$$

В результате из выражения (5) получаем функциональную зависимость напряженности электрического поля $E$ от верхней отрицательно заряженной плоскости дипольного слоя:

$E_{\text {upper plane }}=\left.\left(\frac{P_{S}}{2 \varepsilon_{0}} \frac{z}{\sqrt{z^{2}+r^{2}}}\right)\right|_{0} ^{R}=-\frac{P_{S}}{2 \varepsilon_{0}}\left(1-\frac{z}{\sqrt{z^{2}+R^{2}}}\right)$.

\section{Приложение 2}

Интеграл от суперпозиции напряженности электрических полей от обеих плоскостей есть

$$
\varphi(z)=-\int_{\infty}^{z} \frac{P_{S}}{2 \varepsilon_{0}}\left(\frac{z}{\sqrt{z^{2}+R^{2}}}+\frac{z+d}{\sqrt{(z+d)^{2}+R^{2}}}\right) d z .
$$

Его первообразная

$$
\varphi(z)=\left.\frac{P_{S}}{2 \varepsilon_{0}}\left(\sqrt{(z+d)^{2}+R^{2}}-\sqrt{z^{2}+R^{2}}\right)\right|_{\infty} ^{z} .
$$

Рассмотрим нижний предел функции (П2.2):

$$
\lim _{z \rightarrow \infty} \frac{P_{S}}{2 \varepsilon_{0}}\left(\sqrt{(z+d)^{2}+R^{2}}-\sqrt{z^{2}+R^{2}}\right) .
$$

Преобразуя (П2.3) и раскладывая полученное выражение в ряд Тейлора (пользуясь малостью величины $\frac{2 z d+d^{2}}{z^{2}+R^{2}}$ при $z \rightarrow \infty)$, получаем

$$
\lim _{z \rightarrow \infty} \frac{P_{S}}{2 \varepsilon_{0}} \sqrt{z^{2}+R^{2}}\left(1+\frac{2 z d+d^{2}}{2\left(z^{2}+R^{2}\right)}-1\right)=\frac{P_{S}}{2 \varepsilon_{0}} d .
$$

Подставляя (П2.4) в выражение (П2.2), получаем функциональную зависимость для электрического потенциала любой точки $z$ :

$$
\varphi(z)=\frac{P_{S}}{2 \varepsilon_{0}}\left(\sqrt{(z+d)^{2}+R^{2}}-\sqrt{z^{2}+R^{2}}-d\right) .
$$

\section{Приложение 3}

Представим выражение (5) основного текста в виде

$$
E=\frac{P_{S}}{2 \varepsilon_{0}} \frac{2}{\sqrt{z^{2}+R^{2}}}\left(z-\frac{z+d}{\sqrt{1+\frac{2 z d+d^{2}}{z^{2}+R^{2}}}}\right) .
$$

Раскладывая (П3.1) в ряд Тейлора, после ряда преобразований, при $R \gg z$ получаем

$$
\begin{aligned}
E= & \frac{P_{S}}{2 \varepsilon_{0}} \frac{1}{R}\left(z-\frac{2(z+d) R^{2}}{2 R^{2}+2 z d+d^{2}}\right)=-\frac{P_{S}}{2 \varepsilon_{0}} \\
& \times \frac{2(z+d) R}{2 R^{2}+2 z d+d^{2}}=-\frac{P_{S}}{2 \varepsilon_{0}} \frac{(z+d)}{R} .
\end{aligned}
$$

При условии, что плечо диполя $d \gg z$, из выражения (П3.2) получаем

$$
E=-\frac{P_{S}}{2 \varepsilon_{0}} \frac{d}{R}
$$

Аналогично из уравнения (П2.5) для $R \gg z$ получаем аппроксимацию и для электрического потенциала

$$
\varphi(z)=\frac{P_{S}}{2 \varepsilon_{0}} \sqrt{z^{2}+R^{2}}\left(\sqrt{1+\frac{2 z d+d^{2}}{z^{2}+R^{2}}}-1-\frac{d}{\sqrt{z^{2}+R^{2}}}\right) .
$$

Из (П3.4), используя разложение в ряд Тейлора, получаем

$$
\varphi(z)=\frac{P_{S}}{2 \varepsilon_{0}} \sqrt{z^{2}+R^{2}}\left(1+\frac{2 z_{0} d+d^{2}}{2\left(z^{2}+R^{2}\right)}-1-\frac{d}{\sqrt{z^{2}+R^{2}}}\right) .
$$

Учитывая, что $R \gg z$, преобразуем выражение (П3.5):

$$
\varphi(z)=\frac{P_{S}}{2 \varepsilon_{0}} R\left(\frac{2 z d+d^{2}}{2 R^{2}}-\frac{d}{R}\right) .
$$

Для случая $2 z d \gg d^{2}$ выражение (П3.6) преобразуется в (П3.7):

$$
\varphi\left(z_{0}\right)=\frac{P_{S}}{2 \varepsilon_{0}}\left(\frac{z d}{R}-d\right) .
$$

\section{Приложение 4}

При получении функциональной зависимости для электрического потенциала при $z \gg R$ проинтегрируем выражение (13) основного текста:

$$
\Delta \varphi_{\text {upper plane }}=-\int_{\infty}^{z}-\frac{P_{S} R^{2}}{2 \varepsilon_{0}} \frac{d}{z^{3}} d z=-\left.\frac{P_{S} R^{2} d}{4 \varepsilon_{0} z^{2}}\right|_{\infty} ^{z} .
$$

Таким образом, потенциал от дипольного слоя в любой точке $z$ при $z \gg R$ равен

$$
\varphi(z)=-\frac{P_{S} R^{2}}{4 \varepsilon_{0}} \frac{d}{z^{2}} .
$$




\section{Приложение 5}

Для оценки величины возможных искажений в распределении электрических потенциалов, вносимых пьезоэффектом, воспользуемся выражением для тензора механических напряжений:

$$
S_{i j}=\sum_{k l} C_{i j k l} \varepsilon_{k l}-\sum_{m n} e_{i j m n} E_{m n}
$$

Здесь $S_{i j}$ - тензор механических напряжений, $C_{i j k l}-$ тензор упругих постоянных, $\varepsilon_{k l}-$ тензор деформации $\left(\varepsilon_{k l}=\alpha_{k l} \Delta T\right), e_{i j m n}-$ тензор пьезоэлектрических коэффициентов и $E_{m n}$ - тензор напряженности электрического поля, $\alpha_{j}-$ матрица коэффициентов теплового расширения, $\Delta T$ - изменение температуры исследуемого материала.

В качестве тензора деформации используем представление Коши-Грина:

$$
\varepsilon_{k l}=\frac{1}{2}\left(\frac{\partial u_{k}}{\partial x_{l}}+\frac{\partial u_{l}}{\partial x_{k}}\right),
$$

где $\mathbf{u}$ - вектор, описывающий смещения.

Совместное решение уравнений (П5.1), (П5.2) методом конечных элементов позволяет рассчитать деформации $\varepsilon_{k l}$ как функции температуры $T(t)$. Полученные значения деформации при различных температурах $\varepsilon_{k l}(T(t))$ используем для расчетов величины электрической индукции:

$$
D_{i j}=\sum_{k l} e_{i j k l} \varepsilon_{k l}+\sum_{m n} \varepsilon_{i j m n} E_{m n}
$$

где $\varepsilon_{i j m n}-$ тензор диэлектрической проницаемости пьезоэлектрика.

В предположении отсутствия внешнего поля $\left(\mathbf{E}_{\text {external }}=0\right), \quad$ для $\quad$ величины $\quad$ электрической индукции (П5.3) получаем связь:

$$
\mathbf{D}=\mathbf{P}_{\text {piezo }}
$$

Подставляя значения пьезоэлектрической поляризации $\mathbf{P}_{\text {piezo }}$ в уравнение (19) основного текста статьи, отражающие общую картину распределения потенциала вне зависимости от причины поляризации, получаем величину поверхностного потенциала, вызванного упругими деформациями.

При этом используем:

тензор пьезоэлектрических коэффициентов $e_{i j k l}$ для РZT

$$
\begin{aligned}
& e_{i j k l}= \\
& \left(\begin{array}{cccccc}
0 & 0 & 0 & 17.034 & 0 & 0 \\
0 & 0 & 0 & 0 & 0 & 0 \\
-6.62281 & -6.62281 & 23.24 & 0 & 0 & 0
\end{array}\right)\left[\frac{\mathrm{C}}{\mathrm{m}^{2}}\right],
\end{aligned}
$$

тензор упругих постоянных $C_{i j k l}$ для PZT

$C_{i j k l}=$

$\left(\begin{array}{cccccc}127.2 & 80.21 & 84.67 & 0 & 0 & 0 \\ 80.21 & 127.2 & 84.67 & 0 & 0 & 0 \\ 84.67 & 84.67 & 117.43 & 0 & 0 & 0 \\ 0 & 0 & 0 & 22.98 & 0 & 0 \\ 0 & 0 & 0 & 0 & 22.98 & 0 \\ 0 & 0 & 0 & 0 & 0 & 23.47\end{array}\right)[\mathrm{GPa}]$

и тензор упругих постоянных $C_{i j k l}$ для $\mathrm{Si}$

$$
C_{i j k l}=\left(\begin{array}{cccccc}
166 & 64 & 64 & 0 & 0 & 0 \\
64 & 166 & 64 & 0 & 0 & 0 \\
64 & 64 & 166 & 0 & 0 & 0 \\
0 & 0 & 0 & 80 & 0 & 0 \\
0 & 0 & 0 & 0 & 80 & 0 \\
0 & 0 & 0 & 0 & 0 & 80
\end{array}\right)[\mathrm{GPa}] .
$$

\section{Приложение 6}

Воспользуемся литературными данными о величинах удельных сопротивлений используемых пленок (табл. П6.1).

Таблица П6.1. Величины удельных сопротивлений пироэлектрических пленок, использованные при расчетах двуслойной структуры Si-мембрана/пироэлектрик

\begin{tabular}{c|c}
\hline Пироэлектрик & Удельное сопротивление $\rho_{R}, \Omega \cdot \mathrm{m}$ \\
\hline SBN50 & $10^{7}[37]$ \\
\hline PZT-5H & $10^{7}$ \\
\hline TGS $l$-alanine & $10^{8}[38]$
\end{tabular}

Доминирующим типом шумов при регистрации тепловых изображений является шум Джонсона [39], поэтому для оценки величины теплового шума воспользуемся выражением

$$
V_{N}=\sqrt{4 k T R \Delta f},
$$

где $k-$ постоянная Больцмана, $T-$ температура сенсорно-преобразовательного образца, $R-$ сопротивление сенсорно-преобразовательного образца, $\Delta f-$ эквивалентная частотная полоса пропускания.

Рассчитав величину тепловых шумов, для предельной чувствительности пироэлектрических приемников изображений получаем

$$
\mathscr{R}=\frac{\Delta \phi}{P} .
$$

Результаты расчетов основных параметров сенсорнопреобразовательных материалов, полученных нами для конкретных представителей пироэлектрических пленок, представлены в табл. 4 основного текста статьи. 


\section{Финансирование работы}

Исследование выполнено при финансовой поддержке Российского фонда фундаментальных исследований в рамках научного проекта № 20-38-90125.

\section{Конфликт интересов}

Авторы заявляют, что у них нет конфликта интересов.

\section{Список литературы}

[1] C. Jelen, S.B. Slivken, T. David, G. Brown, M. Razeghi. In: Photodetectors: Materials and Devices III, ed. by G.J. Brown (San Jose, Proc. SPIE, 1998), v. 3287, p. 96-104. DOI: $10.1117 / 12.304470$

[2] O.O. Cellek, S. Ozer, C. Besikci. IEEE J. Quant. Electron., 41 (7), 980 (2005). DOI: 10.1109/JQE.2005.848947

[3] S.U. Eker, Y. Arslan, C. Besikci. Infrared Phys. Technol., 54 (2), 209 (2011). DOI: 10.1016/j.infrared.2010.12.015

[4] L.T. Chee, M. Hooman. Nanophotonics, 7 (1), 1 (2017). DOI: 10.1515/nanoph-2017-0061

[5] В.В. Коротаев, Г.С. Мельников, С.В. Михеев, В.М. Самков, Ю.И. Солдатов. Основы тепловидения (СПб: НИУ ИТМО, СПб., 2012), с. 122.

[6] J.E. Huffman, A.G. Crouse, B.L. Halleck, T.V. Downes. J. Appl. Phys., 72 (1), 273 (1998). DOI: 10.1063/1.352127

[7] N. Sclar. In: Infrared Detectors, ed. by W.L. Wolfe (San-Diego, Proc. SPIE, 1983), v. 0443, p. 11-41. DOI: $10.1117 / 12.937937$

[8] S.M. Birkmann, J. Stegmaier, U. Grözinger, O. Krause. In: High Energy, Optical, and Infrared Detectors for Astronomy III, ed. by D.A. Dorn, A.D. Holland (Marceille, Proc, SPIE, 2008), v. 7021, p. 70210R. DOI: $10.1117 / 12.789103$

[9] S.I. Woods, J.E. Proctor, T.M. Jung, A.C. Carter, J. Neira, D.R. Defibaugh. Appl. Opt., 57 (18), D82 (2018). DOI: $10.1364 / A O .57 .000 D 82$

[10] A. Rogalski. Infrared Phys. Technol., 43 (3-5), 187 (2002). DOI: $10.1016 / \mathrm{S} 1350-4495(02) 00140-8$

[11] G. Eppeldauer, M. Rácz. Appl. Opt., 39 (31), 5739 (2000). DOI: 10.1364/AO.39.005739

[12] H. Yuan, G. Apgar, J. Kim, J. Laquindanum, V. Nalavade. Infrared Technology and Applications XXXIV, ed. by B.F. Andersen, G.F. Fulop, P.R. Norton (Orlando, Proc. SPIE, $2008)$, v. 6940 , p. 69403 C. DOI: $10.1117 / 12.782735$

[13] A. Rogalski. Infrared Phys. Technol., 54(5), 126 (2011). DOI: $10.1016 /$ j.infrared.2010.12.003

[14] I.E. Carranza, J. Grant, J. Gough, R.S. David. IEEE Trans. Terahertz Sci. Technol., 5(6), 892 (2015). DOI: 10.1109/TTHZ.2015.2463673

[15] Y.-Z. Deng, S.-F. Tang, H.-Y. Zeng, Z.-Y. Wu, D.K. Tung. Sensors (Basel), $18(2593), \quad 1 \quad$ (2018). DOI: $10.3390 / \mathrm{s} 18082593$

[16] M.F. Rashman, I.A. Steele, S.D. Bates, D. Copley, S.N. Longmore. Monthly Notices Royal Astronom. Society, 492 (1), 480 (2020). DOI: 10.1093/mnras/stz3497

[17] C. Vedel, J.-L. Martin, J.-L. Ouvrier Buffet. In: Infrared Technology and Applications $X X V$, ed. by B.F. Andersen, M. Strojnik (Orlando, Proc. SPIE, 1999), v. 3698, p. 276283. DOI: $10.1117 / 12.354529$
[18] T. Schimert, D. Ratcliff, J. Brady, S. Ropson, R. Gooch, B. Ritchey, P. McCardel, K. Rachels, M. Wand, M. Weinstein, J. Wyim. In: Unattended Ground Sensor Technologies and Applications, ed. by E.M. Carapezza, D.B. Law, K.T. Stalker (Orlando, Proc. SPIE, 1999), v. 3713, p. 101111. DOI: $10.1117 / 12.357125$

[19] S. Estill, M.R. Brozel. MRS Online Proceed. Library, 299, 27 (1994). DOI: 10.1557/PROC-299-27

[20] C. Hoffman, R. Driggers. Encyclopedia of Optical and Photonic Engineering (Print) - Five Volume Set (CRC Press, Florida, 2015), p. 4088. ISBN: 9781439850978

[21] F.J. Low. J. Optical Society America, 51 (11), 1300 (1961). DOI: $10.1364 /$ JOSA.51.001300

[22] М.А. Тарасов, Л.С. Кузьмин, В.С. Эдельман, Н.С. Каурова, М.Ю. Фоминский, А.Б. Ермаков. Письма в ЖЭТФ, 92, 460 (2010). [M.A. Tarasov, L.S. Kuzmin, V.S. Edelman, N.S. Kaurova, M.Yu. Fominskii, A.B. Ermakov. JETP Lett., 92, 416 (2010). DOI: 10.1134/S0021364010180116]

[23] A. Rogalski. Infrared Detectors: 2nd ed. (CRC Press, Florida, 2020), p. 898. ISBN: 9780367577094

[24] S.K. Holland, R.H. Krauss, G. Laufer. Optical Engineer., 43 (10), 2303 (2004). DOI: 10.1117/1.1782612

[25] C.B. Roundy, R.L. Byer. J. Appl. Phys., 44 (2), 929 (1973). DOI: $10.1063 / 1.1662294$

[26] M.C. Kao, H.Z. Chen, S.L. Yang, Y.C. Chen, P.T. Hsieh, C.C. Yu. Thin. Solid Films, 516 (16), 5518 (2008). DOI: $10.1016 /$ j.tsf.2007.07.020

[27] C. Giebeler, J. Wright, S. Freeborn, N. Conway, T. Chamberlain, P. Clark, M. Schreiter, D. Pitzer, R. Koehle. SENSOR + TEST Conference 2009 (Nürnberg, AMA Service GmbH, 2009), p. 185-189. DOI: 10.5162/irs09/i1.1

[28] C.M. Dudhe, S.B. Nagdeote, C. P. Chaudhari. Taylor \& Francis, Ferroelectrics, 482, 104 (2015). DOI: $10.1080 / 00150193.2015 .1057080$

[29] W.R. Cook,jr. Piezoelectric, Pyroelectric, and Related Constants (Springer-Verlag, Berlin, Heidelberg, Berlin, 1994), p. 543. ISBN: 978-3-540-55065-5

[30] S.T. Liu, R.B. Maciolek. J. Electron. Mater., 4 (1), 91 (1975). DOI: $10.1007 / \mathrm{BF} 02657838$

[31] H.V. Alexandru, C. Berbecaru, F. Stanculescu, L. Pintilie, I. Matei, M. Lisca. Sensors Actuators A: Phys., 113 (3), 387 (2004). DOI: 10.1016/j.sna.2004.03.046

[32] W.A. Tiller. The Science of Crystallization Macroscopic Phenomena and Defect Generation (Cambridge University Press, California, 1992), p. 520. ISBN: 9780521388283

[33] S. Yarlagadda, H.W. Chan, H. Lee. J. Intelligent Mater. Systems Structures, $6(6), 757 \quad$ (1995). DOI: $10.1177 / 1045389 X 9500600603$

[34] J. Ouyang. Enhanced Piezoelectric Performance of Printed PZT Films on Low Temperature Substrates (Rochester, Rochester Institute of Technology, 2017)

[35] T.A. Germer, J.C. Zwinkels, B.K. Tsai. Spectrophotometry: Accurate Measurement of Optical Properties of Materials (Amsterdam, Academic Press, 2014), v. 46, p. 533. ISBN: 9780123860224 
[36] W.R. Cook,jr. Piezoelectric, Pyroelectric, and Related Constants (Springer-Verlag, Berlin, Heidelberg, Berlin, 1994), p. 543. ISBN: 978-3-540-55065-5

[37] L. Zhang, R. Barrett, P. Cloetens, C. Detlefs, M. Sanchez del Rio. J. Synchrotron Rad., 21, 507 (2014). DOI: $10.1107 / \mathrm{S} 1600577514004962$

[38] Y. Wu, G. Caoa. J. Mater. Res., 15(7), 1583 (2000). DOI: $10.1557 / J M R .2000 .0227$

[39] M. Vollmer, K.-P. Mollmann. Infrared Thermal Imaging Fundamentals, Research and Applications (Wiley-VCH, 2018), p. 794. ISBN: 978-3-527-41351-5 